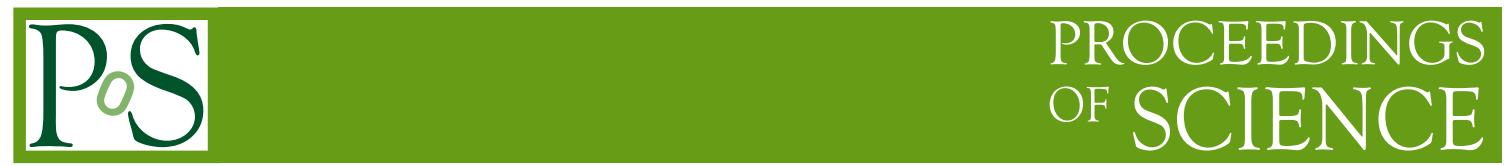

\title{
Collider probes of real triplet scalar dark matter
}

\author{
Yong $\mathbf{D u}{ }^{a, b, *}$ \\ ${ }^{a}$ Amherst Center for Fundamental Interactions, Department of Physics, University of Massachusetts, \\ Amherst, MA 01003, USA \\ ${ }^{b}$ CAS Key Laboratory of Theoretical Physics, Institute of Theoretical Physics, \\ Chinese Academy of Sciences, Beijing 100190, China \\ E-mail: yongdu@umass.edu
}

We study the real triplet model in which the neutral component of the triplet is a dark matter candidate and the charge components are long-lived. The long-lived triplet particles will result in disappearing charged tracks when produced at colliders. Utilizing this disappearing charged track signature from the decay of the charged components, we study exclusion limits from the LHC and a prospective $100 \mathrm{TeV} p p$ collider. We also investigate the dark matter candidate and obtain constraints from present and future dark matter direct detection experiments. We conclude that XENON20T could cover almost the entire parameter space of this model except for a vanishingly small portal coupling.

The Eighth Annual Conference on Large Hadron Collider Physics-LHCP2020

25-30 May, 2020

online

${ }^{*}$ Speaker 


\section{Introduction}

The evidence for the existence of dark matter is tremendous, and the latest measurement from the Planck satellite gives a dark matter energy density of $\Omega_{\mathrm{DM}} h=0.1198 \pm 0.0012$ [1], where $h$ is the Hubble constant in units of $100 \mathrm{~km} /(\mathrm{s} \cdot \mathrm{Mpc})$. It has been known for a while that the Standard Model (SM) could not account for this dark matter relic density and new physics is therefore needed.

Among many dark matter proposals, Weakly Interacting Massive Particles (WIMPs) have gain significant attention in the past decades due to their mass range, which is of $O(10 \mathrm{GeV}-10 \mathrm{TeV})$, and its possible connection to other fundamental questions including the hierarchy problem, the baryon asymmetry of the Universe, etc. The WIMP scenario have also been inspiring many experiments looking for dark matter signature either directly through measuring the recoil energy of the nuclei from dark matter scattering, or indirectly by looking for energetic SM particles from dark matter annihilation in nearby dark matter halo, or by producing dark matter particles directly at colliders.

In our recent work [2], we study the real triplet model obtained by extending the SM scalar sector with a real triplet that transforms as $(1,3,0)$ under the SM gauge group. We focus on the scenario in which the neutral component of the real triplet is a WIMP dark matter candidate and the charged components are long-lived particles resulting in disappearing charged tracks (DCTs) once produced at colliders. We study constraints on this model from both the DCT signature at colliders and dark matter direct detection.

\section{Model setup}

The Lagrangian for the real triplet model is

$$
\begin{aligned}
& \mathcal{L}=\left(D_{\mu} H\right)^{\dagger}\left(D^{\mu} H\right)+\left(D_{\mu} \Sigma\right)^{\dagger}\left(D^{\mu} \Sigma\right)-V(H, \Sigma), \\
& D_{\mu} \Sigma=\partial_{\mu} \Sigma+i g_{2}\left[W_{\mu}, \Sigma\right], \quad W_{\mu}=W_{\mu}^{a} \tau^{a} / 2
\end{aligned}
$$

with $\tau^{a}$ the Pauli matrices, the SU(2) Higgs doublet $H$ and the real triplet scalar $\Sigma$ given by

$$
H=\left(\begin{array}{c}
G^{+} \\
\frac{1}{\sqrt{2}}\left(v+h+i G^{0}\right)
\end{array}\right), \quad \Sigma=\frac{1}{2}\left(\begin{array}{cc}
\Sigma^{0} & \sqrt{2} \Sigma^{+} \\
\sqrt{2} \Sigma^{-} & -\Sigma^{0}
\end{array}\right),
$$

and $v \simeq 246 \mathrm{GeV}$ being the Higgs vacuum expectation value (VEV). The scalar potential can be expressed as

$$
V(H, \Sigma)=-\mu^{2} H^{\dagger} H+\lambda_{0}\left(H^{\dagger} H\right)^{2}-\frac{1}{2} \mu_{\Sigma}^{2} F+\frac{b_{4}}{4} F^{2}+\frac{a_{2}}{2} H^{\dagger} H F,
$$

with $F=\left(\Sigma^{0}\right)^{2}+2 \Sigma^{+} \Sigma^{-}$. Note that the portal coupling $a_{2}$ measures the interacting strength between the doublet and the real triplet. Upon imposing a discrete $Z_{2}$ symmetry and assigning a $Z_{2}$-odd parity for $\Sigma$ only, $\Sigma^{0}$ becomes stable and our dark matter candidate. At tree level, $\Sigma^{0, \pm}$ are degenerated mass eigenstates. Higher-loop electroweak radiative corrections break the degeneracy and lead to $[3,4]$

$$
\Delta m=m_{\Sigma^{ \pm}}-m_{\Sigma^{0}}=166 \mathrm{MeV}(\mathrm{NLO})+\text { a few } \mathrm{MeV}(\mathrm{NNLO}),
$$




\begin{tabular}{l|llll}
\hline \hline Annihilation & \multicolumn{4}{|c}{ Coannihilation } \\
\hline \hline$* \Sigma^{0} \Sigma^{0} \rightarrow W^{ \pm} W^{\mp}$ & $\Sigma^{0} \Sigma^{ \pm} \rightarrow f \bar{f}^{\prime}$ & $* \Sigma^{ \pm} \Sigma^{\mp} \rightarrow f \bar{f}$ & $* \Sigma^{ \pm} \Sigma^{\mp} \rightarrow h \gamma$ & $\Sigma^{ \pm} \Sigma^{\mp} \rightarrow v \bar{v}$ \\
$* \Sigma^{0} \Sigma^{0} \rightarrow Z Z$ & $\Sigma^{0} \Sigma^{ \pm} \rightarrow W^{ \pm} Z$ & $* \Sigma^{ \pm} \Sigma^{\mp} \rightarrow W^{ \pm} W^{\mp}$ & $* \Sigma^{ \pm} \Sigma^{\mp} \rightarrow h h$ & $\Sigma^{ \pm} \Sigma^{ \pm} \rightarrow W^{ \pm} W^{ \pm}$ \\
$* \Sigma^{0} \Sigma^{0} \rightarrow h h$ & $\Sigma^{0} \Sigma^{ \pm} \rightarrow W^{ \pm} \gamma$ & $* \Sigma^{ \pm} \Sigma^{\mp} \rightarrow Z Z$ & $\Sigma^{ \pm} \Sigma^{\mp} \rightarrow Z \gamma$ & \\
$* \Sigma^{0} \Sigma^{0} \rightarrow f \bar{f}$ & $* \Sigma^{0} \Sigma^{ \pm} \rightarrow W^{ \pm} h$ & $* \Sigma^{ \pm} \Sigma^{\mp} \rightarrow Z h$ & $\Sigma^{ \pm} \Sigma^{\mp} \rightarrow \gamma \gamma$ & \\
\hline \hline
\end{tabular}

Table 1: Annihilation and coannihilation processes related to our dark matter relic density calculation, where $f$ stands for all massive fermions in the SM and $v=v_{e}, v_{\mu}, v_{\tau}$. Processes starting with an asterisk $(*)$ are $a_{2}$ dependent.

making the decay process $\Sigma^{ \pm} \rightarrow \Sigma^{0} \pi^{ \pm}$kinematically allowed with a branching ratio of $98 \%$. Note that since this mass splitting is very close to the rest mass of pions, as a result, pions in the final state would be too soft to be reconstructed, and experimentally, DCTs would be observed upon production of $\Sigma^{ \pm}$at colliders. The LHC is very sensitive to the DCT signature and can therefore be used to explore this model as will be discussed below.

\section{Strategy}

To utilize the DCT signature to constrain this model, we firstly reproduce the ATLAS $13 \mathrm{TeV}$ result in Ref. [5], which looks for a long-lived chargino with the DCT signature, to validate our setup. We then generalize our code for the real triplet model for the LHC, the future high-luminosity LHC (HL-LHC), as well as a possible future $100 \mathrm{TeV} p p$ collider to obtain collider constraints on this model. On the other hand, due to the smallness of the mass splitting $\Delta m$, contributions to dark matter relic density from coannihilation processes listed in Table 1 needs to be included. In addition, as was previously found in the "Minimal Dark Matter" scenario, the real triplet needs to be about $2.5 \mathrm{TeV}$ to saturate the observed dark matter relic density [3], such that SM particles can be effectively taken as massless and the resulting long-range interactions, also known as the Sommerfeld effect, needs to be taken into account to correctly obtain prediction of dark matter relic density from the real triplet model. Based on consideration above, our results are shown in Figure 1 below and will be discussed in the next section.

\section{Results}

Using the DCT signature and taking coannihilaiton and the Sommerfeld effect into account, our results are shown in Figure 1. The left plot in Figure 1 corresponds to LHC exclusion limits from the DCTs where different colorful regions are obtained from the application of different luminosity. We find that, depending on the lifetime of the charged triplet whose variation is a result of the inclusion of two-loop electroweak radiative corrections in Eq. (5), the LHC has presently excluded a real triplet lighter than $\sim 275 \mathrm{GeV}$ and the HL-LHC would improve that to $\sim 590$ (745) GeV for $\mathcal{L}=300(3000) \mathrm{fb}^{-1}$. Moreover, a prospective $100 \mathrm{TeV} p p$ collider would improve the exclusion limit even further to about $3 \mathrm{TeV}$ depending on the pile up effects.

On the dark matter side, assuming that the real triplet saturates the dark matter relic density, we find the triplet needs to be at lightest $\sim 2.5 \mathrm{TeV}$ as implied by the green region in the middle 

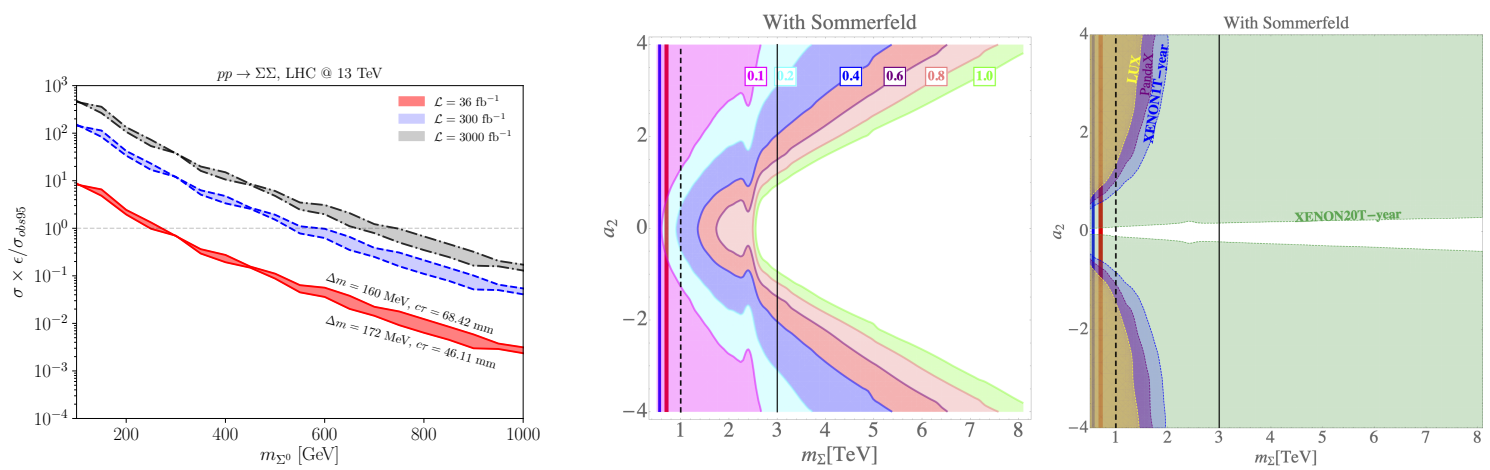

Figure 1: Left panel: Exclusion limits from the LHC with indicated luminosity from the DCT signature. The colorful regions above the horizontal dashed line have been (would have been) excluded by the current (future high-luminosity) LHC. Middle panel: The parameter space that can explain current dark matter relic density with the inclusion of the Sommerfeld effect. Numbers in boxes on the curves correspond to the fractions of the real triplet contributing to the total dark matter relic density. The vertical bands (lines) corresponds to the exclusion limit from the LHC (a future $100 \mathrm{TeV} p p$ collider). Right panel: Exclusion limits from dark matter direct detection from LUX [6], Panda-X [7] and XENON1T [8] with the same vertical bands or lines as in the middle plot.

plot. We further note that the real triplet needs to contribute at least about $10 \%$ of the total dark matter relic density if no DCT signature is observed at the LHC and/or the HL-LHC, as can be seen from comparing the vertical blue band with the purple and cyan regions in the middle plot. Given WIMP dark matter is severely constrained by direct detection experiments, we also impose these constraints onto the real triplet model parameter space. We find XENON1T currently gives the most stringent constraint and has excluded a real triplet lighter than $\sim 2 \mathrm{TeV}$ when the magnitude of the portal coupling $a_{2}$ is of $O(1)$. This can be clearly seen from the blue region in the last plot of Figure 1. Also note that in the future, as shown by the green region in the last plot, XENON20T would be able to cover almost the entire parameter space of this model except when $a_{2}$ is vanishingly small. This deficiency is due to the fact that, when $a_{2}$ is tiny, dark matter-nuclei interacting strength will be very weak, and as a result, the recoil energy of the xenon nuclei would be too small to cause any observable effects.

\section{Summary}

The real triplet have been studied in detail and the DCT signature from the decay of $\Sigma^{ \pm}$ has been used to obtain exclusion limits on this model from current and future hadron colliders. We find that, depending on the lifetime of the charged triplet, currently the LHC excludes a real triplet lighter than $\sim 275 \mathrm{GeV}$ and the HL-LHC would improve that limit to $\sim 590$ (745) GeV for $\mathcal{L}=300(3000) \mathrm{fb}^{-1}$. A possible future $100 \mathrm{TeV} p p$ collider would improve that limit even further to about $3 \mathrm{TeV}$ depending on the pile up effects. On the other hand, XENON1T gives the most stringent constraint on the real triplet model from dark matter direct detection experiments, and presently excludes a real triplet lighter than about $2 \mathrm{TeV}$ when the portal coupling $a_{2}$ is of $O(1)$. In the future, XENON20T would be able to cover almost the entire parameter space of the real triplet model except when $a_{2}$ is vanishingly small. 


\section{References}

[1] Planck collaboration, N. Aghanim et al., Planck 2018 results. VI. Cosmological parameters, 1807.06209.

[2] C.-W. Chiang, G. Cottin, Y. Du, K. Fuyuto and M. J. Ramsey-Musolf, Collider Probes of Real Triplet Scalar Dark Matter, 2003.07867.

[3] M. Cirelli, N. Fornengo and A. Strumia, Minimal dark matter, Nucl. Phys. B753 (2006) 178-194, [hep-ph/0512090].

[4] M. Ibe, S. Matsumoto and R. Sato, Mass Splitting between Charged and Neutral Winos at Two-Loop Level, Phys. Lett. B 721 (2013) 252-260, [1212 . 5989].

[5] ATLAS collaboration, M. Aaboud et al., Search for long-lived charginos based on a disappearing-track signature in pp collisions at $\sqrt{s}=13 \mathrm{TeV}$ with the ATLAS detector, JHEP 06 (2018) 022, [1712.02118].

[6] LUX collaboration, D. S. Akerib et al., Results from a search for dark matter in the complete LUX exposure, Phys. Rev. Lett. 118 (2017) 021303, [1608. 07648].

[7] PandaX-II collaboration, X. Cui et al., Dark Matter Results From 54-Ton-Day Exposure of PandaX-II Experiment, Phys. Rev. Lett. 119 (2017) 181302, [1708.06917].

[8] XENON collaboration, E. Aprile et al., Dark Matter Search Results from a One Ton-Year Exposure of XENON1T, Phys. Rev. Lett. 121 (2018) 111302, [1805. 12562]. 